\title{
Photothermal therapy with AuNRs and EGFRmAb-AuNRs inhibits subcutaneous transplantable hypopharyngeal tumors in nude mice
}

\author{
YINGYING ZHANG $^{1 *}$, JIAYAN HE ${ }^{2 *}$, YU WANG $^{2}$, JINYA WEN $^{2}$, YINGYING ZOU $^{1}$, \\ ZHIHONG YANG ${ }^{1}$ and XIAOGUANG $\mathrm{HE}^{2}$ \\ ${ }^{1}$ Department of Pathology and Pathophysiology, School of Basic Medical Science, \\ Kunming Medical University, Kunming, Yunnan 650500; ${ }^{2}$ Department of Head and Neck Surgery, \\ The First Affiliated Hospital of Kunming Medical University, Kunming, Yunnan 650032, P.R. China
}

Received May 8, 2015; Accepted August 8, 2018

DOI: $10.3892 /$ ijo.2018.4559

\begin{abstract}
The present study aimed to investigate the effects of photothermal therapy with gold nanorods (AuNRs) or epidermal growth factor receptor monoclonal antibody-conjugated AuNRs (EGFRmAb-AuNRs) on hypopharyngeal carcinoma $(\mathrm{HC})$ in nude mice. In addition, the associated signaling pathways were explored. Briefly, a subcutaneous transplantable hypopharyngeal tumor model was established in nude mice injected with FaDu human HC cells. A total of 70 nude mice were randomly divided into seven groups, each of which received a different treatment. Mice were treated with AuNRs, locally or through intravenous injection, whereas EGFRmAb or EGFRmAb-AuNRs were only administered locally. Near infrared spectroscopy (NIR) was also applied for plasmonic photothermal therapy (PPTT). The growth curve and the inhibitory rate for tumor growth were used to evaluate the effects of each treatment. Flow cytometry and the terminal-deoxynucleotidyl transferase dUTP nick end labeling assay were adopted to detect apoptosis of cells in the transplanted tumors. Reverse transcription-quantitative polymerase chain reaction and western blotting were used to determine the mRNA and protein expression levels of target genes, respectively. Local treatment with AuNRs + NIR or EGFRmAb significantly inhibited tumor growth, and EGFRmAb conju-
\end{abstract}

Correspondence to: Professor Xiaoguang He, Department of Head and Neck Surgery, The First Affiliated Hospital of Kunming Medical University, 295 Xichang Road, Kunming, Yunnan 650032, P.R. China

E-mail: xghe1018@163.com

*Contributed equally

Key words: gold nanorods, photothermal therapy, epidermal growth factor receptor monoclonal antibody, phosphatidylinositol-3-kinase/AKT serine/threonine kinase signaling pathway, DNA damage signaling pathway gation further increased the inhibitory effects. Furthermore, there was a significant increase in apoptosis of tumor cells in the AuNRs + NIR, EGFRmAb and EGFRmAb-AuNRs + NIR groups; treatment with EGFRmAb-AuNRs + NIR induced the highest apoptotic effect. Mechanistic studies indicated that EGFRmAb-AuNRs + NIR may inhibit tumors through the AKT serine/threonine kinase (Akt) and DNA damage signaling pathways. In the AKT pathway, the mRNA and protein expression levels of phosphatase and tensin homolog were increased, whereas the expression levels of Akt and glycogen synthase kinase $3 \beta$ were decreased. In the DNA damage signaling pathway, the mRNA and protein expression levels of ATR serine/threonine kinase, checkpoint kinase 1 and p53 were enhanced, whereas phosphorylated-p53 protein expression was reduced. The present findings indicated that AuNRs + NIR inhibited HC tumor growth, and conjugating EGFRmAb to AuNRs further enhanced the inhibitory effects. EGFRmAb conjugation may increase the antitumor effects of AuNRs-induced PPTT by downregulating the phosphatidylinositol-3-kinase/Akt pathway and upregulating the DNA damage pathway. These findings may provide novel insights into tumor-targeting PPTT in vivo.

\section{Introduction}

Hypopharyngeal carcinoma (HC) is a malignant tumor of the head and neck, which has the highest mortality rate in all patients with head and neck cancer. Hypopharyngeal squamous cell carcinoma (HNSCC) is the most common pathological type of $\mathrm{HC}$, which is closely associated with smoking, drinking, air pollution, long-term exposure to toxic chemicals, human papilloma virus, sex hormones and other factors (1); however, its etiology remains unclear. Patients with $\mathrm{HC}$ are usually asymptomatic, and it is commonly diagnosed at the middle-late stage. The 5-year survival rate for $\mathrm{HC}$ is $\sim 40 \%$ and has not improved over the last two decades (2); therefore, the development of novel therapeutic strategies is required to improve the survival rate.

Gold nanoparticles (AuNPs) have specific physical and chemical properties; they are easy to make and exhibit good 
stability and biocompatibility, as well as strong surface plasticity. In addition, AuNPs can be conjugated to antibodies, carbohydrates, DNA/RNA and other factors, which may specifically target tumor cells. These features make AuNPs promising tools for plasmonic photothermal therapy (PPTT) and tumor-targeting therapy (3). PPTT involves the rapid conversion of light into heat by plasmonic nanoparticles that are targeted to a tumor, thus inducing hyperthermia-induced cell death. These nanoparticles can be passively targeted using the enhanced permeability and retention effect, or they can be actively targeted using proteins, peptides and other small molecules. AuNPs have been reported to induce plasmonic photothermal cell death, and are able to monitor associated molecular alterations through time-dependent surface-enhanced Raman spectroscopy within a single cell (4). Gold nanorods (AuNRs) are a specific type of AuNP, which have been applied to treat head and neck tumors. AuNRs have two characteristic surface plasmon resonance (SPR) absorbance peaks. The transverse SPR is $\sim 520 \mathrm{~nm}$ and is a characteristic of all AuNPs, whereas the long SPR (LSPR) is a variable of its aspect ratio and can therefore be manually tuned. When the aspect ratio is 3.9, the LSPR is $800 \mathrm{~nm}$, which is in the middle of the near infrared region (i.e. 700-900 nm). Near infrared spectroscopy (NIR) can penetrate deeply into living tissues with few fluorescence effects on the biological content in the tissue; therefore, it is considered beneficial for PPTT of deep tumors. AuNR-mediated PPTT is able to selectively kill tumor cells labeled with AuNRs, without damaging the surrounding normal cells. Therefore, AuNR-induced PPTT may be an emerging strategy for cancer therapy (4).

AuNRs have a strong affinity with antibodies, proteins and DNA fragments, and the resulting conjugates can target tumor cells more specifically. It has been reported that epidermal growth factor receptor (EGFR) is highly expressed in various types of human malignant cancer, including colorectal cancer, breast cancer, pancreatic cancer, and head and neck squamous cell carcinoma (5). Therefore, EGFR is considered an important biotherapy target in numerous types of solid tumor. EGFR monoclonal antibody-conjugated AuNRs (EGFRmAb-AuNRs) have been generated, and the apoptotic potential of EGFRmAb-AuNRs has been assessed in Hep-2 cells in vitro and in vivo (6). However, few studies have focused on the effects of EGFRmAb-AuNRs on HNSCC; therefore, further investigation is required.

The phosphatidylinositol-3-kinase (PI3K)/AKT serine/threonine kinase (Akt) pathway is one of the most important pathways activated by EGFR $(7,8)$. During tumor development, it is critical for increasing tumor cell proliferation, inhibiting apoptosis, enhancing angiogenesis, and promoting invasion and metastasis $(9,10)$. Akt, which is also termed protein kinase $\mathrm{B}$, is the key effector downstream of PI3K (11). In response to external stimulation, PI3K is activated, which in turn transforms Akt into phosphorylated (p)-Akt (12).

Endogenous and exogenous factors, including abnormal metabolites and ionizing radiation, induce DNA damage $(13,14)$. Usually, cells initiate the DNA damage response against such damage, and when the damage is repairable, it can be fixed by the internal repair system (15). However, if the damage is too severe and cannot be recovered, apoptosis is triggered (16).
Previously, we synthesized AuNRs using the cetyltrimethylammonium bromide system and conjugated EGFRmAb to AuNRs. Furthermore, it was revealed that 293T and FaDu human HC cell lines overexpress EGFR on the surface of the cell membrane. Subsequently, it was revealed that EGFRmAb-AuNRs selectively kill FaDu cells under suitable NIR, thus suggesting that EGFRmAb may have a synergistic role and may significantly improve the efficiency of AuNR-induced PPTT (17). However, in vitro data may not correctly reflect what occurs in vivo, since tumor growth is a complex process that depends on the interaction between tumor cells and their microenvironment. Therefore, the present study aimed to investigate and verify the effects of AuNRs and EGFRmAb-AuNRs PPTT on subcutaneous transplantable hypopharyngeal tumors in nude mice. In addition, the roles of the PI3K/Akt and DNA damage signaling pathways were explored in AuNR-mediated apoptosis.

\section{Materials and methods}

Cell lines and cell culture. The FaDu human pharynx squamous cell carcinoma cell line was purchased from the Shanghai Cell Bank of Chinese Academy of Sciences (Shanghai, China). Cell lines were cultured in RPMI-1640 medium supplemented with $10 \%$ fetal bovine serum (FBS), $1 \%$ penicillin-streptomycin and $1 \%$ L-glutamine (all from Gibco; Thermo Fisher Scientific, Inc., Waltham, MA, USA) at $37^{\circ} \mathrm{C}$ in a humidified atmosphere containing $5 \% \mathrm{CO}_{2}$.

Preparation of AuNRs and conjugation with EGFRmAb. AuNRs were obtained from Kunming Institute of Precious Metals (Kunming, China). EGFRmAb was purchased from Sigma-Aldrich; Merck KGaA (Darmstadt, Germany). AuNRs and EGFRmAb-AuNRs used for animal studies were prepared as previously described (6). Briefly, EGFRmAb was dissolved in HEPES buffer (20 nmol; pH 7.4) and mixed with AuNRs solution to generate a solution with an optical density of 0.8 at $800 \mathrm{~nm}$. Subsequently, the synthetic EGFRmAb-AuNRs $(0.1 \mathrm{nmol} / \mathrm{l})$ were examined under a transmission electron microscope (JEM 1010; JEOL Ltd., Tokyo, Japan) to determine the mean value of the long and transverse diameter of the particles.

Generation of a HC model in nude mice. A total of 70 healthy female BALB/C(nu/nu) nude mice (age, 5-6 weeks; weight, 19-22 g) were purchased from Hunan Silaike Jingda Laboratory Animal Co. Ltd. (Changsha, China). All mice were housed in a standard pathogen-free facility under constant temperature $\left(25 \pm 2^{\circ} \mathrm{C}\right)$ and humidity $(45-50 \%)$ at the experimental animal center of Kunming Medical University. All experimental procedures were conducted in accordance with the institutional guidelines for the care and use of laboratory animals, and the present study was approved by the Institutional Animal Care and Use Committee of Kunming Medical University.

FaDu cells in the logarithmic growth phase were collected and resuspended in PBS (Gibco; Thermo Fisher Scientific, Inc.) to make a single cell suspension. Subsequently, $200 \mu \mathrm{l}$ PBS solution containing $1 \times 10^{7}$ cells was injected into the back of nude mice using $1 \mathrm{ml}$ sterile syringes. Following injection of FaDu cells, HC tumors began to develop after 7 days. The 
mice were randomly divided into seven groups; each group contained 10 mice. When the tumor size reached $\sim 200 \mathrm{~mm}^{3}$, a total of 3 weeks after injection, each group was treated differently. In the control group [normal saline (NS).it], 0.9\% NS was injected directly into tumors. In the AuNRs.it group, AuNRs were injected directly into tumors. In the AuNRs. iv + NIR group, mice were treated with intravenous injection of AuNRs, and received NIR laser irradiation $24 \mathrm{~h}$ post-injection. Mice in the NIR group received local irradiation only, without any injection. In the AuNRs.it + NIR group, AuNRs were injected directly into tumors, followed by NIR treatment $24 \mathrm{~h}$ post-injection. In the EGFRmAb.it group, EGFRmAb was injected directly into tumors. In the EGFRmAb-AuNRs. it + NIR group, EGFRmAb-AuNRs was injected directly into tumors, and NIR treatment was performed $24 \mathrm{~h}$ post-injection. The total injection volume was $200 \mu \mathrm{l}$ for all reagents (0.9\% N.S, EGFRmAb, AuNRs and EGFRmAb-AuNRs). In the present study, the wavelength of NIR was $808 \mathrm{~nm}$, the power was $5.0 \mathrm{~W} / \mathrm{cm}^{2}$, and the duration was $6 \mathrm{~min}$. The laser-transmitting end was placed $2 \mathrm{~cm}$ away from the tumors to ensure the laser spot covered the entire tumor.

Following treatment, the mice were observed every other day and body weight, food intake, sleep, activity and defecation were recorded. The long diameter (a), short diameter (b) and height (c) of the tumors were also recorded every other day. Tumor volume was calculated using the following equation: $\mathrm{V}=\mathrm{a} \times \mathrm{b} \times \mathrm{c}$; tumor growth curves were generated accordingly. A total of 3 days after treatment, the mice were sacrificed using cervical dislocation and tumors were dissected from the body. The inhibition rate for tumor weight was calculated as follows: Average tumor weight in the control group - average tumor weight in the experimental group) $x 100 \%$. The inhibition rate for tumor volume was calculated as follows: Average tumor volume in the control group - average tumor volume in the experimental group) x $100 \%$. Subsequently, five tumors were taken randomly from each group and stored in $4 \%$ polyformaldehyde solution at room temperature for terminal-deoxynucleotidyl transferase dUTP nick end labeling (TUNEL) staining. The other five tumors in each group were maintained in PBS at room temperature for flow cytometry, reverse transcription-quantitative polymerase chain reaction (RT-qPCR) and western blot analysis.

Apoptosis analysis using flow cytometry. Tumor tissues (100 mg) were digested with $500 \mu 10.25 \%$ pancreatin-EDTA solution at $37^{\circ} \mathrm{C}$ for $10 \mathrm{~min}$, after which, $500 \mu \mathrm{l}$ RPMI-1640 medium supplemented with $10 \%$ FBS was added to terminate the reaction. The resulting solution was centrifuged at $1,750 \mathrm{xg}$ for $5 \mathrm{~min}$, and the pellet was resuspended in PBS to make a single cell suspension $\left(5 \times 10^{5}\right.$ cells $\left./ \mathrm{ml}\right)$. Annexin V-fluorescein isothiocyanate (FITC) and propidium iodide (PI) were used to label apoptotic and necrotic cells, according to the manufacturer's protocol (cat. no. 556547; BD Biosciences, Franklin Lakes, NJ, USA). The stained cells were detected using flow cytometry and were analyzed with FlowJo 7.6.2 (FlowJo, LLC, Ashland, OR, USA). Surviving cells exhibited very low fluorescence, whereas apoptotic cells were stained with Annexin V-FITC and exhibited strong green fluorescence, and necrotic cells were stained with Annexin V-FITC and PI, thus exhibiting green and red fluorescence.
Apoptosis detection using TUNEL assay. Tumor samples were fixed and sectioned. Subsequently, a TUNEL assay (cat. no. E607175; Sangon Biotech Co., Ltd., Shanghai, China) was performed to determine the presence of apoptotic cells in situ. The TUNEL assay identifies apoptotic cells by the terminal deoxynucleotidyl transferase-mediated addition of DIG-labeled deoxyridine triphosphate nucleotides (DIG-dUTPs) to the 3'-OH end of DNA strand breaks. The TUNEL-labeled cells are subsequently visualized using anti-DIG-biotin, streptavidin-biotin complex (SABC) and DAB.

Briefly, tumor tissues were fixed with $10 \%$ formalin at room temperature for $12 \mathrm{~h}$, and formalin-fixed tissues were embedded in paraffin. Subsequently, sections $(5 \mu \mathrm{m})$ were prepared and transferred onto glass slides; the formalin-fixed, paraffin-embedded tumor sections were deparaffinized and rehydrated in a coplin jar. Prepared slides were incubated with freshly made $3 \% \mathrm{H}_{2} \mathrm{O}_{2}$ in the dark for $10 \mathrm{~min}$ at room temperature, and were then washed three times with $0.01 \mathrm{M}$ Tris-buffered saline (TBS; $5 \mathrm{~min} /$ wash). Subsequently, the slides were treated with $20 \mu \mathrm{g} / \mathrm{ml}$ proteinase $\mathrm{K}$ without DNase at $37^{\circ} \mathrm{C}$ for $5 \mathrm{~min}$, and were then rinsed three times in $0.01 \mathrm{M}$ TBS ( $2 \mathrm{~min} / \mathrm{wash}$ ). Each slide was treated with $20 \mu \mathrm{l}$ working solution containing $1 \mu \mathrm{l}$ TdT, $1 \mu \mathrm{l}$ DIG-dUTP and $18 \mu \mathrm{l}$ labeling buffer at $37^{\circ} \mathrm{C}$ for $2 \mathrm{~h}$, after which, $50 \mu \mathrm{l}$ sealing solution was applied to each slide for $30 \mathrm{~min}$ at room temperature. Subsequently, $50 \mu \mathrm{l}$ anti-DIG-biotin antibody (1:100) was added to each slide, and the slides were incubated at $37^{\circ} \mathrm{C}$ for $30 \mathrm{~min}$. SABC (1:100) and DAB were used sequentially to visualize the signal. Apoptotic cells were stained brown and were observed under a light microscope.

The sections were viewed under a light microscope (400x magnification) to identify TUNEL staining. Nine fields were randomly selected in each slide, and the number of TUNEL-positive cells was determined as the average number of stained cells in the observed fields. Image Pro Plus 6.0 software (Media Cybernetics, Inc., Rockville, MD, USA) was used to determine the light intensity of TUNEL staining. Similarly, nine fields were randomly selected and observed in each slide. The light intensity of TUNEL-positive cells in a slide was determined as the average number of the nine fields.

mRNA expression detection using RT-qPCR. Tumor tissues (100 mg) were used for total RNA extraction using $800 \mu \mathrm{l}$ TRIzol ${ }^{\circledR}$ lysis buffer (Invitrogen; Thermo Fisher Scientific, Inc.). Total mRNA was initially extracted with chloroform, and then with isopropanol, and was finally dissolved in $20 \mu \mathrm{l} \mathrm{DEPC}$ water. Subsequently, first-strand cDNA was synthesized using the RevertAid ${ }^{\mathrm{TM}}$ First Strand cDNA Synthesis kit (Thermo Fisher Scientific, Inc.) according to the manufacturer's protocol. Gene-specific primers used for RT-qPCR were designed with Beacon Designer 7.90 software (Premier Biosoft International, Palo Alto, CA, USA) and synthesized by Invitrogen; Thermo Fisher Scientific, Inc. Genes were amplified using a SYBR-Green master mix and the ABI 7300 Real-Time PCR system (Applied Biosystems; Thermo Fisher Scientific, Inc.). $\beta$-actin was used as an internal control. The RT-qPCR reaction protocol was as follows: After denaturation at $95^{\circ} \mathrm{C}$ for $30 \mathrm{sec}$, the mixture was amplified for 45 cycles. Each amplification cycle consisted of 5 min of denaturation at $95^{\circ} \mathrm{C}$ to activate the enzyme, $1 \mathrm{~min}$ of denaturation at $95^{\circ} \mathrm{C}, 1 \mathrm{~min}$ of annealing at $58^{\circ} \mathrm{C}$ and $1 \mathrm{~min}$ at 

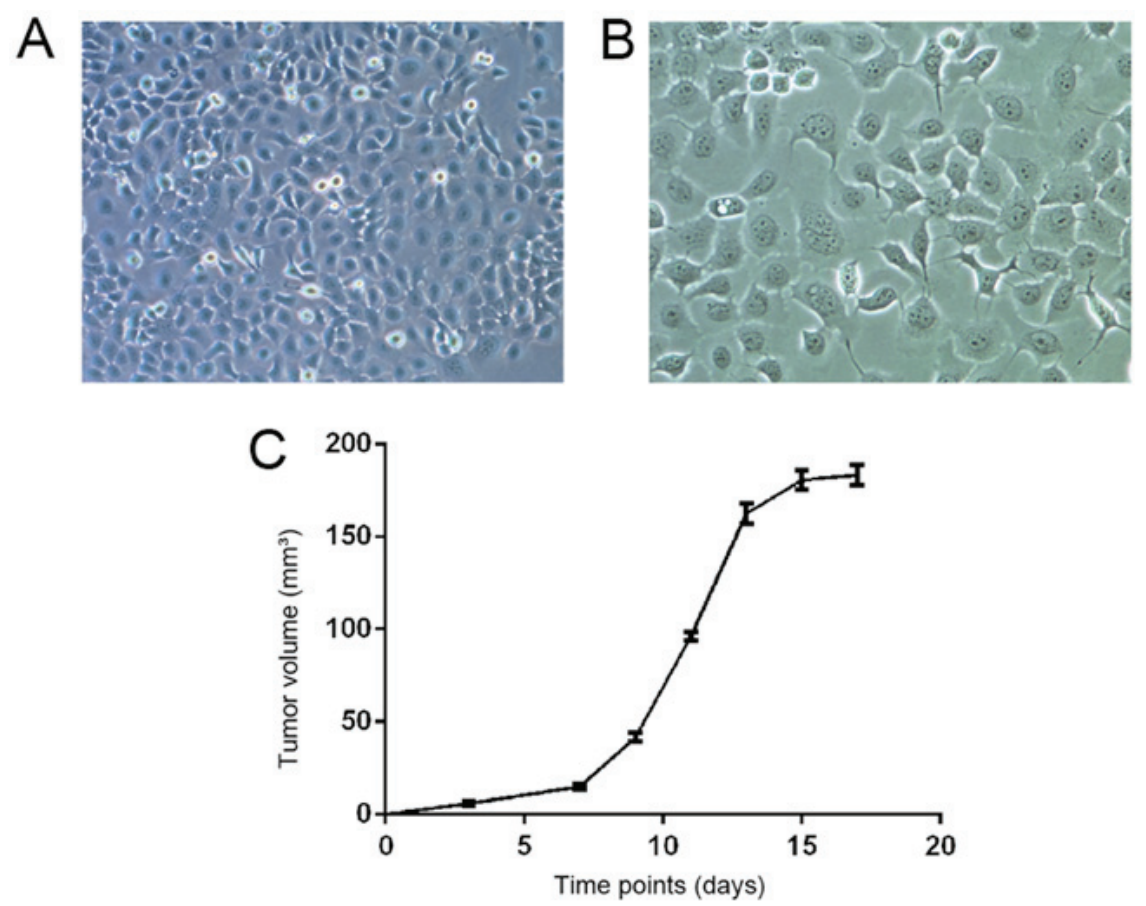

Figure 1. FaDu cells were injected into nude mice for tumor development. (A and B) FaDu cell morphology under a light microscope: (A) Magnification, x100; (B) magnification, x200. (C) FaDu cells formed tumors in nude mice. The tumor growth curve is indicated. Tumors reached $200 \mathrm{~mm}^{3} 17$ days post-injection.

$72^{\circ} \mathrm{C}$. The final extension step was $15 \mathrm{~min}$ at $72^{\circ} \mathrm{C}$. Each set of experiments was repeated three times. The relative expression of detected genes was calculated using the $2^{-\Delta \Delta \mathrm{Cq}}$ method (18). The primer sequences were as follows: $\beta$-actin, forward, 5'-TATGGAATCCTGTGGCATC-3', reverse, 5'-GTGTTGGCA TAGAGGTCTT-3'; Akt, forward, 5'-TATTGTGAAGGA GGGTTG-3', reverse, 5'-ATTCTTGAGGAGGAAG TAG-3'; ATR serine/threonine kinase (ATR), forward, 5'-AGAGACG GAATGAAGATT-3', reverse, 5'-GAGATTAGATTATTGA GGACTT-3'; checkpoint kinase 1 (Chk1), forward, 5'-GTGTGA ATGACAACTACTG-3', reverse, 5'-TGCTGTATGTTCGGT ATT-3'; glycogen synthase kinase $3 \beta$ (GSK3 $\beta$ ), forward, 5'-TAATCTGGTGCTGGACTA-3', reverse, 5'-CTGATACATA TACAACTTGACAT-3'; p53, forward, 5'-AGTATTTGG ATGACAGAA-3', reverse, 5'-ATGTAGTTGTAGTGGATG-3'; and phosphatase and tensin homolog (PTEN), forward, 5'-ACGAACTGGTGTAATGATA-3' and reverse, 5'-GTCTCT GGTCCTTACTTC-3'.

Protein expression detection using western blotting. Tumor tissues $(100 \mathrm{mg})$ were used for total protein extraction. Samples were lysed in $800 \mu 1$ radioimmunoprecipitation assay buffer containing protease inhibitors (Beyotime Institute of Biotechnology, Haimen, China). Protein concentrations were subsequently determined using a bicinchoninic acid (Beyotime Institute of Biotechnology) assay at $562 \mathrm{~nm}$, according to the manufacturer's protocol. Equal amounts of protein $(70 \mu \mathrm{g})$ were denatured in $1 \mathrm{X}$ loading buffer at $100^{\circ} \mathrm{C}$ for $10 \mathrm{~min}$ and were separated by $4 \%$ SDS-PAGE. Subsequently, proteins were transferred to polyvinylidene fluoride membranes. The resulting membranes were blocked with $5 \%$ bovine serum albumin (Beijing Solarbio Science \& Technology Co. Ltd., Beijing, China) for $1 \mathrm{~h}$ at room temperature. The membranes were then individually incubated overnight at $4^{\circ} \mathrm{C}$ with primary antibodies against $\beta$-actin (cat. no. T40104), pan-Akt (cat. no. 4691), p-Akt (cat. no. 4060), p-GSK3 $\beta$ (cat. no. 9322), GSK3 $\beta$ (cat. no. 5676), PTEN (cat. no. 9552), p-ATR (cat. no. 2853), ATR (cat. no. 2790), p-Chk1 (cat. no. 2348), Chk1 (cat. no. 2360), p-p53 (cat. no. 9286) and p53 (cat. no. 2527). Subsequently, the membrane was incubated with horseradish peroxidase-conjugated secondary antibodies [1:1,000; M21001 (anti-mouse) and M21002 (anti-rabbit), Shanghai Abmart Co., Ltd.] for $2 \mathrm{~h}$ at room temperature. Images of the membranes were captured with a Bio-Rad GelDock system (Bio-Rad Laboratories, Inc., Hercules, CA, USA) and the band intensity was determined with ImageJ version 1.8.0 software (National Institutes of Health, Bethesda, MD, USA). The majority of primary antibodies were purchased from Cell Signaling Technology, Inc. (Danvers, MA, USA) and were diluted to 1:1,000, whereas anti- $\beta$-actin antibodies were obtained from Shanghai Abmart Co., Ltd. (Shanghai, China) and were diluted to 1:2,000.

Statistical analysis. SPSS 18.0 software (SPSS, Inc., Chicago, IL, USA) was used to analyze the data. Quantitative data from triplicate experiments were presented as the means \pm standard deviation. One-way analysis of variance was used to compare multiple groups and the least significant difference test was applied to make comparisons between two groups. $\mathrm{P}<0.05$ was considered to indicate a statistically significant difference.

\section{Results}

Construction of a HC model in nude mice. $\mathrm{HC}$ is one of the most severe malignant tumors in the upper aerodigestive tract. To investigate the effects of AuNRs on tumor growth in vivo, FaDu HC cells (Fig. 1A and B) were injected into the backs of nude mice. There was no significant difference in body weight prior to FaDu cell injection and no mice succumbed during 
Table I. Tumor volume before and after treatment $(n=10)$.

\begin{tabular}{|c|c|c|c|}
\hline \multirow[b]{2}{*}{ Group } & \multirow{2}{*}{$\frac{\text { Before treatment }}{\text { Tumor volume }\left(\mathrm{mm}^{3}\right)}$} & \multicolumn{2}{|c|}{ After treatment } \\
\hline & & Tumor volume $\left(\mathrm{mm}^{3}\right)$ & Tumor weight (g) \\
\hline NS.it & $179.00 \pm 3.30$ & $268.57 \pm 24.98^{a}$ & $4.93 \pm 0.21$ \\
\hline Au.it & $179.66 \pm 2.00$ & $259.37 \pm 22.70^{\mathrm{a}}$ & $5.07 \pm 0.91$ \\
\hline Au.iv + NIR & $180.39 \pm 1.46$ & $252.76 \pm 10.85^{\mathrm{a}}$ & $4.53 \pm 0.21$ \\
\hline NIR & $178.73 \pm 3.01$ & $264.64 \pm 17.63^{\mathrm{a}}$ & $4.60 \pm 0.27$ \\
\hline Au.it + NIR & $179.53 \pm 2.04$ & $80.99 \pm 4.18^{\mathrm{a}-\mathrm{e}}$ & $3.18 \pm 0.31^{\mathrm{a}-\mathrm{d}}$ \\
\hline EGFRmAb.it & $180.18 \pm 0.83$ & $91.95 \pm 5.93^{\mathrm{a}, \mathrm{b}}$ & $3.70 \pm 0.10^{\mathrm{a}}$ \\
\hline EGFRmAb-Au.it + NIR & $179.24 \pm 2.48$ & $54.12 \pm 5.33^{\mathrm{a}, \mathrm{b}, \mathrm{e}-\mathrm{g}}$ & $1.23 \pm 0.21^{\mathrm{a}, \mathrm{d}-\mathrm{f}}$ \\
\hline
\end{tabular}

Data are presented as the means \pm standard deviation. ${ }^{a} \mathrm{P}<0.05$ compared with the groups before treatment; ${ }^{\text {b }}<0.05$ compared with the NS group; ${ }^{\mathrm{C}} \mathrm{P}<0.05$ compared with the Au.it group; ${ }^{\mathrm{d}} \mathrm{P}<0.05$ compared with the $\mathrm{Au}$.iv + NIR group; ${ }^{\mathrm{e}} \mathrm{P}<0.05$ compared with the NIR group; ${ }^{\mathrm{f}} \mathrm{P}<0.05$ compared with the Au.it + NIR group; ${ }^{\mathrm{g}} \mathrm{P}<0.05$ compared with the EGFRmAb.it group. Au, gold nanorods; EGFRmAb, epidermal growth factor monoclonal antibody; it, intratumoral; iv, intravenous; NIR, near infrared spectroscopy; N.S., normal saline.
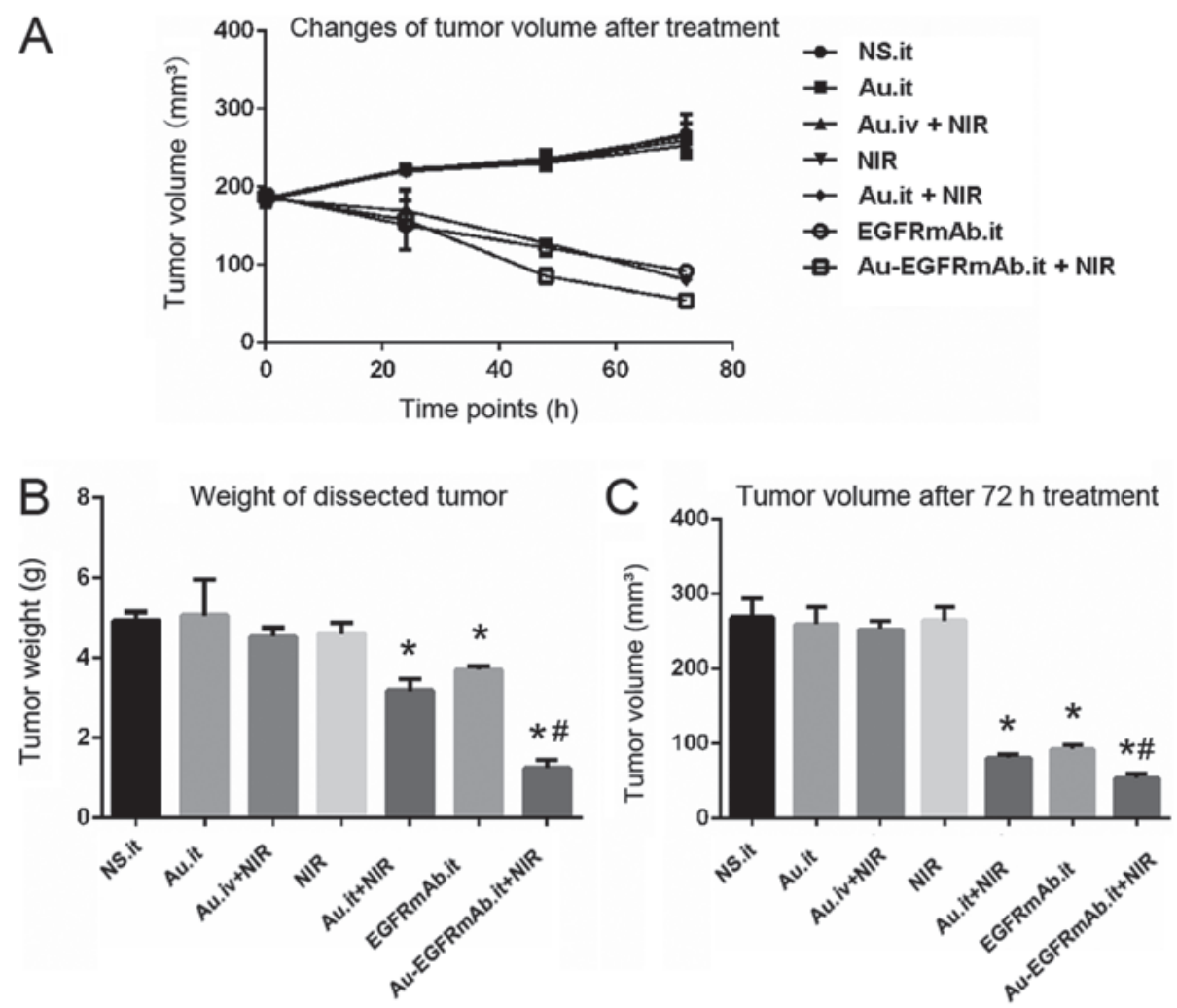

Figure 2. EGFRmAb increases the tumor inhibitory effects of AuNRs on nude mice. (A) Tumor growth curves following treatment. (B) Weight of the dissected tumor. After treatment, mice in the control, Au.it, Au.iv + NIR and NIR groups exhibited similar tumor growth. (C) Tumor volume after $72 \mathrm{~h}$ treatment. The tumor volume in mice treated with Au.it + NIR, EGFRmAb.it and Au-EGFRmAb.it + NIR was significantly decreased. "P<0.05 compared with the NS.it group; ${ }^{*} \mathrm{P}<0.05$ compared with the Au.it + NIR and EGFRmAb groups. Au/AuNRs, gold nanorods; EGFRmAb, epidermal growth factor monoclonal antibody; it, intratumoral; iv, intravenous; NS, normal saline; NIR, near infrared spectroscopy.

tumor development. Furthermore, there was no redness or swelling at the site of injection. After 7 days of inoculation, subcutaneous nodules with a diameter of $\sim 7 \mathrm{~mm}$ developed, thus suggesting the successful establishment of a HC model.

After FaDu cell injection, tumor growth was observed and recorded every other day. As shown in Fig. 1C, the tumor gradually grew from the first week, whereas tumor growth was more rapid in the second week.
AuNR-mediated photothermal therapy effectively reduces tumor growth in vivo, and the inhibitory effect is enhanced by EGFRmAb conjugation. Tumor size was measured before treatment, and no significant difference was observed among the seven groups (Table I). In the control group, tumor volume was $179.00 \pm 3.30 \mathrm{~mm}^{3}$ prior to injection and reached $268.57 \pm 24.98 \mathrm{~mm}^{3} 72 \mathrm{~h}$ post-injection. Tumors grew from $179.66 \pm 2.00$ to $259.37 \pm 22.70 \mathrm{~mm}^{3}$ in the AuNRs.it group, from 

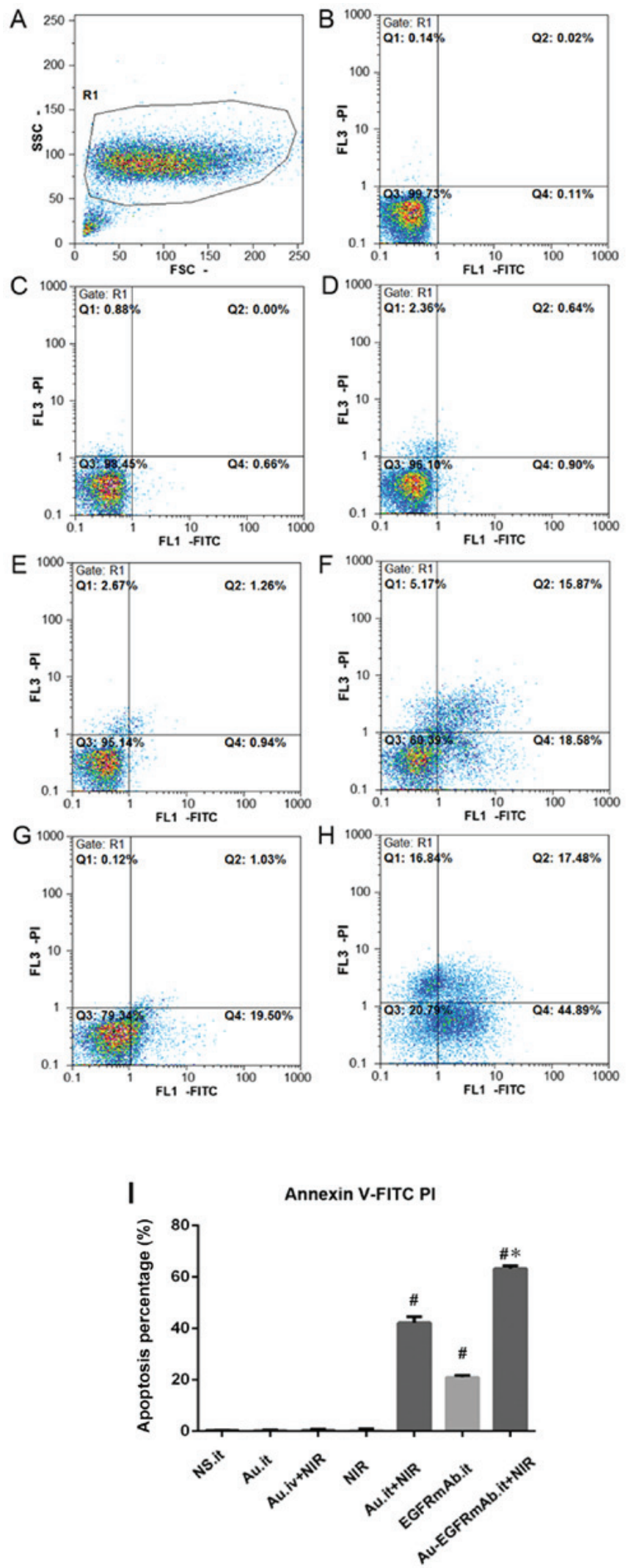

Figure 3. EGFRmAb increases apoptosis induced by AuNRs plasmonic photothermal therapy. (A) Forward and side scatter gating. (B) NS.it group; (C) Au.it group; (D) Au.iv + NIR group; (E) NIR group; (F) Au.it + NIR group; (G) EGFRmAb.it group; (H) Au-EGFRmAb.it + NIR group. (I) Increased apoptosis was observed in tumor cells from the Au.it + NIR, EGFRmAb.i and Au-EGFRmAb.it + NIR-treated mice. ${ }^{~} \mathrm{P}<0.05$ compared with the NS.it group; ${ }^{*} \mathrm{P}<0.05$ compared with the Au.it + NIR and EGFRmAb.it groups. $\mathrm{Au} / \mathrm{AuNRs}$, gold nanorods; EGFRmAb, epidermal growth factor monoclonal antibody; FITC, fluorescein isothiocyanate; it, intratumoral; iv, intravenous; NS, normal saline; NIR, near infrared spectroscopy; PI, propidium iodide.
Table II. Comparison of apoptotic rate in tumor tissues from each group $(n=5)$.

\begin{tabular}{lc}
\hline Group & Apoptotic rate $(\%)$ \\
\hline NS.it & $0.29 \pm 0.14$ \\
Au.it & $0.38 \pm 0.21$ \\
Au.iv + NIR & $0.47 \pm 0.29$ \\
NIR & $0.57 \pm 0.34$ \\
Au.it + NIR & $42.23 \pm 2.33^{\mathrm{a}-\mathrm{d}}$ \\
EGFRmAb.it & $20.97 \pm 0.72^{\mathrm{a}}$ \\
EGFRmAb-Au.it + NIR & $63.30 \pm 1.11^{\mathrm{a}, \mathrm{d}-\mathrm{f}}$
\end{tabular}

Data are presented as the means \pm standard deviation. ${ }^{\text {a }}<0.05$ compared with the NS group; ${ }^{\mathrm{b}} \mathrm{P}<0.05$ compared with the Au.it group; ${ }^{\mathrm{C}} \mathrm{P}<0.05$ compared with the Au iv + NIR group; ${ }^{\mathrm{d}} \mathrm{P}<0.05$ compared with the NIR group; ${ }^{\mathrm{e}} \mathrm{P}<0.05$ compared with the Au. it + NIR group; ${ }^{\mathrm{f}} \mathrm{P}<0.05$ compared with the EGFRmAb.it group. Au, gold nanorods; EGFRmAb, epidermal growth factor monoclonal antibody; it, intratumoral; iv, intravenous; NIR, near infrared spectroscopy; N.S., normal saline.

$178.73 \pm 3.01$ to $264.64 \pm 17.63 \mathrm{~mm}^{3}$ in the NIR group, and from $180.39 \pm 1.46$ to $252.76 \pm 10.85 \mathrm{~mm}^{3}$ in the AuNRs.iv + NIR group. There were no obvious differences in tumor growth between the control group and the three aforementioned experimental groups ( $\mathrm{P}>0.05$; Fig. 2 and Table I). Furthermore, tumors were dissected from nude mice $72 \mathrm{~h}$ post-treatment and tumor weight was measured. As shown in Fig. 2 and Table I, tumor weight was similar among the control, AuNRs.it, NIR and AuNRs.iv + NIR groups. These results suggested that AuNRs alone, NIR alone and AuNRs.iv + NIR had no effect on tumor growth.

The present study also determined whether local application of AuNRs + NIR inhibited tumor growth. Notably, tumor volume decreased by $54.89 \%$ (from $179.53 \pm 2.04$ to $\left.80.99 \pm 4.18 \mathrm{~mm}^{3}\right)$ in the AuNRs.it + NIR group $(\mathrm{P}<0.05)$. In addition, the tumor weight was $35.5 \%$ less compared with in the control group $(\mathrm{P}<0.05)$. In the EGFRmAb.it group, tumor size was reduced by $48.97 \%$ from $180.18 \pm 0.83$ to $91.95 \pm 5.93 \mathrm{~mm}^{3}$ $(\mathrm{P}<0.05)$, and the tumor weight was $24.95 \%$ less than the weight in the control group $(\mathrm{P}<0.05)$. EGFRmAb was conjugated to AuNRs and injected into the tumor, followed by NIR. The results indicated that EGFRmAb-ANRS.it + NIR presented significantly stronger tumor inhibition efficiency than AuNRs.it + NIR or EGFRmAb $(\mathrm{P}<0.05)$. The EGFRmAb-AuNRs.it + NIR mice exhibited the smallest tumor volume $\left(54.12 \pm 5.33 \mathrm{~mm}^{3}\right)$ and the inhibition rate was highest (69.8\%). The tumor weight was $1.23 \pm 0.21 \mathrm{~g}$, and the inhibition rate was $75.1 \%$ (Fig. 2 and Table I).

EGFRmAb conjugation increases AuNRs-mediated apoptosis. The reduced tumor size and weight may be due to increased cell apoptosis or decreased proliferation. Therefore, flow cytometry was performed to detect apoptosis of tumor cells in each group. The results suggested that apoptotic rates in the AuNRs.it, NIR and AuNRs.iv + NIR groups were not markedly different compared with the control group. However, mice treated with AuNRs.it + NIR or EGFRmAb alone exhibited markedly increased apoptosis of tumor cells compared with in the control group ( $\mathrm{P}<0.05$; Fig. 3 and Table II). There 

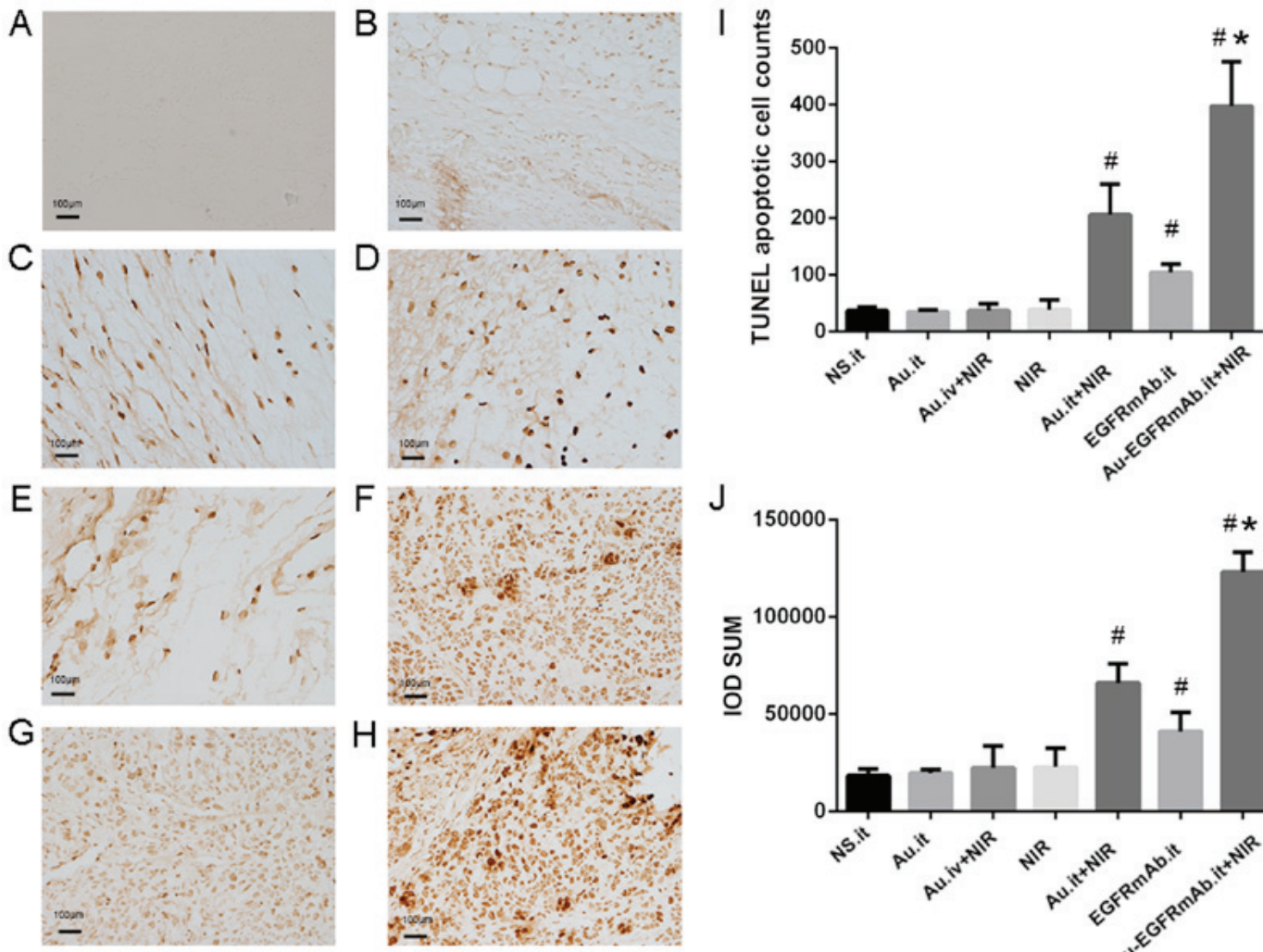

Figure 4. EGFRmAb-Au.it + NIR treatment exhibited the strongest apoptotic effect in TUNEL assays. Tumor tissues were stained with the TUNEL assay kit. (A) PBS control without TUNEL; (B) NS.it group; (C) Au.it group; (D) Au.iv + NIR group; (E) NIR group; (F) Au.it + NIR group; (G) EGFRmAb. it group; (H) EGFRmAb-Au.it + NIR group. (I) Number of apoptotic cells in each group. (J) IOD values of positively stained cells in each group. ${ }^{\#} \mathrm{P}<0.05$ compared with the NS.it group; ${ }^{*} \mathrm{P}<0.05$ compared with the Au.it + NIR and EGFRmAb.it groups. Au, gold nanorods; EGFRmAb, epidermal growth factor monoclonal antibody; IOD, integrated optical density; it, intratumoral; iv, intravenous; NS, normal saline; NIR, near infrared spectroscopy; TUNEL, terminaldeoxynucleotidyl transferase dUTP nick end labeling.

was no significant difference between the AuNRs.it + NIR and EGFRmAb groups. Furthermore, apoptosis was significantly increased in the EGFRmAb-AuNRs.it + NIR group compared with in the AuNRs.it + NIR or EGFRmAb groups $(\mathrm{P}<0.05$; Fig. 3 and Table II). These results suggested that EGFRmAb may act synergistically with AuNRs and may trigger apoptosis following NIR treatment.

The number of apoptotic cells was counted and the integrated optical density (IOD) of positively stained cells was calculated by individual researchers that were blinded to the groups. As shown in Fig. 4, apoptotic cells were stained brown. In the control group, few apoptotic cells were observed (37.25 \pm 6.24 cells), and the total IOD was $18,413.80 \pm 3,268.79$. Consistent with the flow cytometry data, TUNEL assays indicated that the number of apoptotic cells and the IOD value in the AuNRs.it, NIR and AuNRs.iv + NIR groups were similar to the control group $(\mathrm{P}>0.05)$. However, the number of apoptotic cells in the AuNRs.it + NIR, EGFRmAb.it and EGFRmAb-AuNRs.it + NIR groups were 5.5, 2.8 and 10.7 times higher than in the control group; these differences were all statistically significant $(\mathrm{P}<0.05$; Fig. 4 and Table III). Furthermore, the IOD values in these three groups were significantly higher than in the control group $(\mathrm{P}<0.05$; Fig. 4 and Table III). In all test groups, the most marked apoptotic effect was detected in mice treated with EGFRmAb-AuNRs. it + NIR, further confirming the synergistic effect of EGFRmAb on AuNR-induced PPTT.
EGFRmAb-AuNRs PPTT inhibits the PI3K/Akt signaling pathwaybyincreasing PTEN expression. SinceAuNR-mediated PPTT inhibited tumor growth in nude mice, and EGFRmAb enhanced the effect, the present study further investigated the underlying molecular mechanism. The PI3K/Akt pathway is activated in various types of cancer by EGFR, and it has been reported that EGFR is overexpressed in HC (17). Akt and GSK $3 \beta$ are key factors of the PI3K/Akt pathway; therefore, their mRNA expression levels were detected using RT-qPCR. The results indicated that the mRNA expression levels of Akt and GSK3 $\beta$ were similar in mice treated with AuNRs.it, AuNRs. iv + NIR and NIR compared with in the control group ( $\mathrm{P}>0.05$; Fig. 5A and B). Compared with in the control group, the mRNA expression levels of Akt and GSK3 $\beta$ were significantly reduced in mice treated with AuNRs.it + NIR, EGFRmAb. it or EGFRmAb-AuNRs.it + NIR ( $<<0.01$; Fig. 5A and B). Since PTEN is a negative regulator of the PI3K/Akt pathway, the present study aimed to determine whether an increase in PTEN may contribute to reduced activation of the PI3K/Akt pathway. The results of a RT-qPCR analysis indicated that, compared with in the control group, PTEN mRNA expression was not markedly altered in the AuNRs.it, AuNRs.iv + NIR and NIR groups $(\mathrm{P}>0.05)$, but was obviously increased in the AuNRs.it + NIR, EGFRmAb.it and EGFRmAb-AuNRs. it + NIR groups $(\mathrm{P}<0.05$; Fig. $5 \mathrm{C})$. In addition, conjugation of EGFRmAb to AuNRs (EGFRmAb-AuNRs.it + NIR) markedly reduced the mRNA expression levels of Akt and GSK3 $\beta$, 

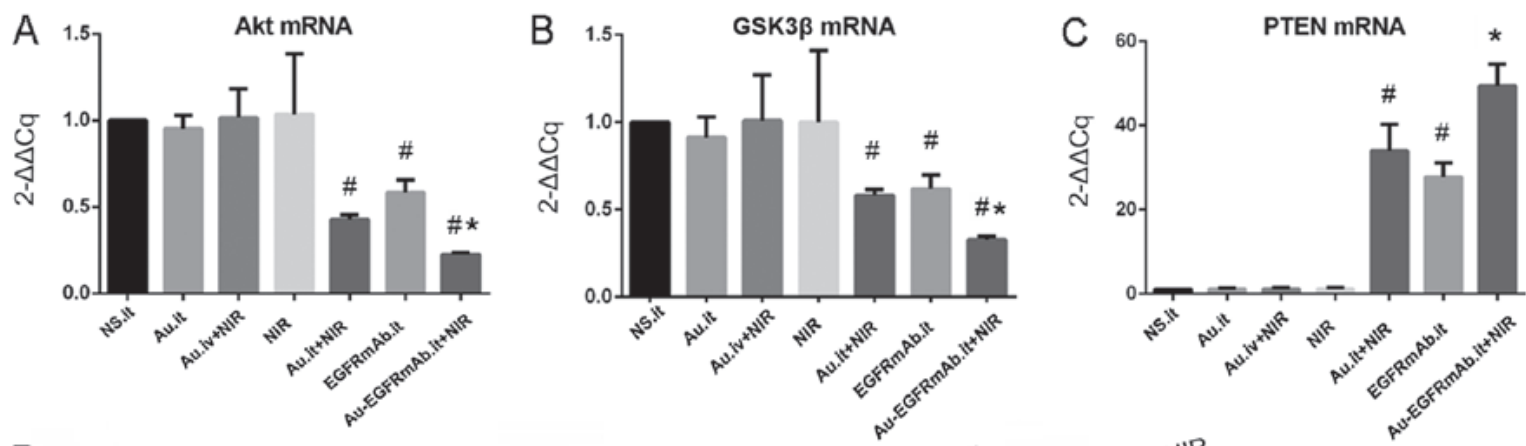

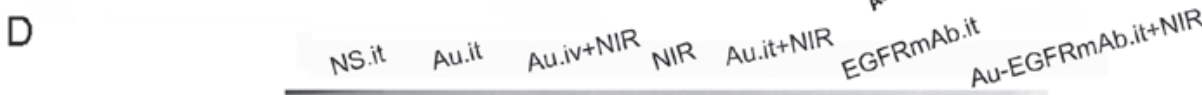

$60 \mathrm{kDa}$

pan-akt

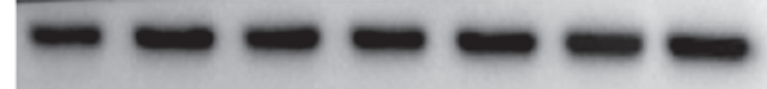

p-akt

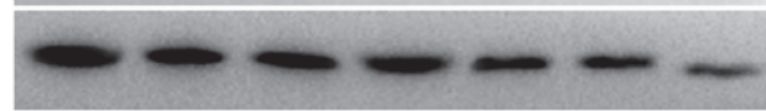

$60 \mathrm{kDa}$

(ser473)

GSK3 $\beta$

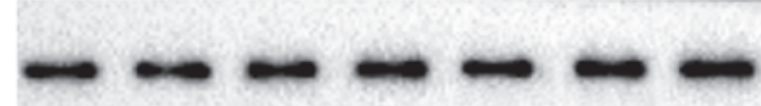

$46 \mathrm{kDa}$

p-GSK3 $\beta$

(ser9)

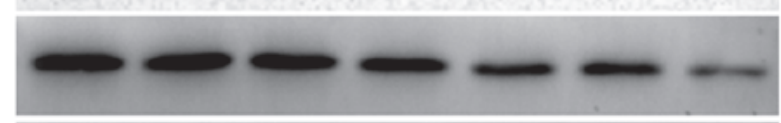

$46 \mathrm{kDa}$

PTEN

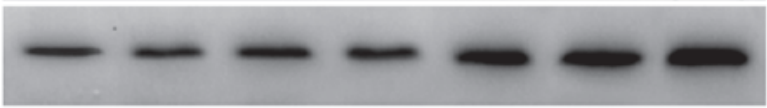

\section{$54 \mathrm{kDa}$}

$\beta$-actin

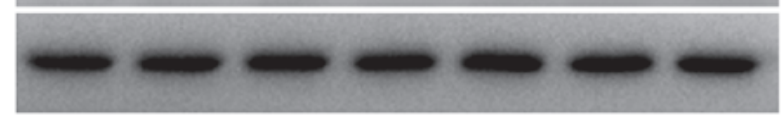

\section{$43 \mathrm{kDa}$}
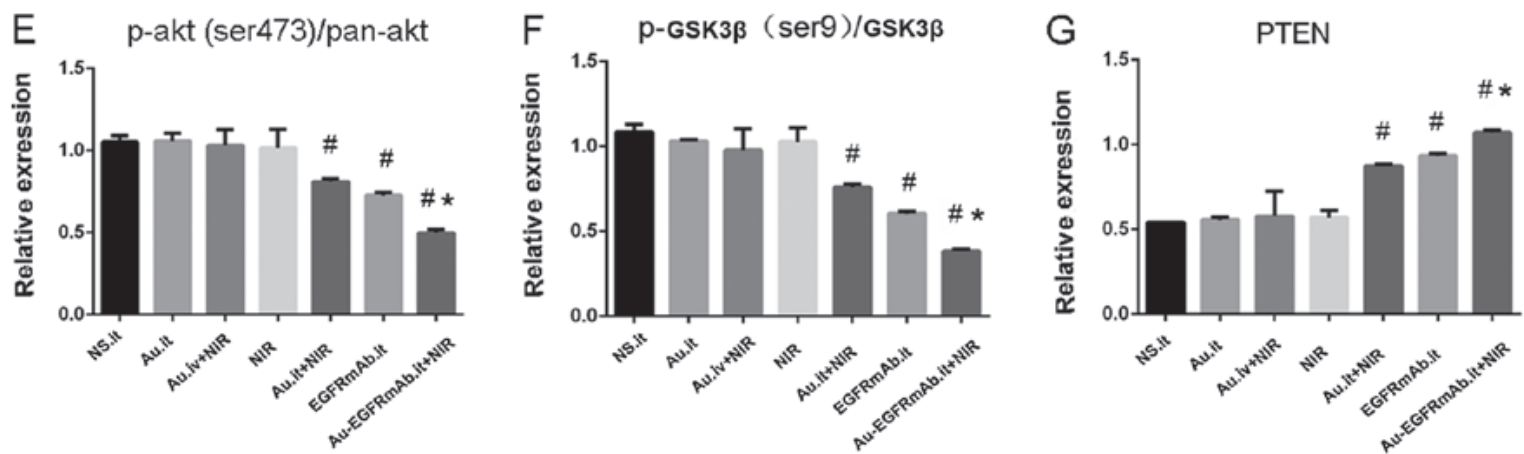

Figure 5. AuNRs plasmonic photothermal therapy inhibits the phosphatidylinositol-3-kinase/Akt pathway, and EGFRmAb conjugation enhances the effect. (A-C) mRNA expression levels of Akt, GSK3 and PTEN. mRNA expression of each sample was detected by reverse transcription-quantitative polymerase chain reaction. The expression data of each group were normalized to the control group (N.S.it). (D-G) Protein expression levels of p-Akt, p-GSK3 $\beta$ and PTEN. Relative protein expression levels are presented in the bar charts. $\beta$-actin was used as a control. ${ }^{~} \mathrm{P}<0.05$ compared with the NS.it group; $\mathrm{P}<0.05$ compared with the Au.it + NIR and EGFRmAb.it groups. All experiments were repeated at least three times. Akt, AKT serine/threonine kinase; Au, gold nanorods; EGFRmAb, epidermal growth factor monoclonal antibody; GSK3 $\beta$, glycogen synthase kinase $3 \beta$; it, intratumoral; iv, intravenous; NS, normal saline; NIR, near infrared spectroscopy; p, phosphorylated; PTEN, phosphatase and tensin homolog.

and increased PTEN expression compared with in the AuNRs. it + NIR or EGFRmAb.it groups $(\mathrm{P}<0.05$; Fig. 5A-C).

To further verify the effects of AuNRs.it + NIR,EGFRmAb. it and EGFRmAb-AuNRs.it + NIR on p-Akt, p-GSK3 $\beta$ and PTEN expression, western blotting was conducted to detect their protein levels. As shown in Fig. 5D-G, the protein expression levels of p-Akt, p-GSK3 $\beta$ and PTEN were not altered in the AuNRs.it, NIR and AuNRs.iv + NIR groups compared with in the control group ( $\mathrm{P}>0.05$; Fig. 5D-G). However, treatment with AuNRs.it + NIR, EGFRmAb. it and EGFRmAb-AuNRs.it + NIR significantly reduced
p-Akt and p-GSK3 $\beta$ protein expression, but increased PTEN expression ( $\mathrm{P}<0.01$; Fig. 5D-G). Furthermore, p-Akt and p-GSK3 $\beta$ were much lower, and PTEN was much higher, in the EGFRmAb-AuNRs.it + NIR group compared with in the AuNRs.it + NIR or EGFRmAb.it groups ( $\mathrm{P}<0.05$; Fig. 5D-G). These results suggested that EGFRmAb may synergistically interact with AuNRs to inhibit the PI3K/Akt pathway, potentially through increasing PTEN expression.

AuNR-mediated PPTT activates the DNA damage signaling pathway, and EGFRmAb conjugation enhances the effect. The 
Table III. Apoptotic cells and IOD values, as detected using terminal-deoxynucleotidyl transferase dUTP nick end labeling assays $(n=5)$.

\begin{tabular}{lcr}
\hline Group & Number of apoptotic cells & IOD sum \\
\hline NS.it & $37.25 \pm 6.24$ & $18,413.80 \pm 3,268.79$ \\
Au.it & $35.00 \pm 3.37$ & $19,634.14 \pm 1,860.90$ \\
Au.iv + NIR & $37.25 \pm 12.09$ & $22,441.31 \pm 11,318.44$ \\
NIR & $39.00 \pm 16.85$ & $22,774.24 \pm 9,644.58$ \\
Au.it + NIR & $206.50 \pm 53.17^{\mathrm{a}-\mathrm{e}}$ & $66,185.23 \pm 9,857.81^{\mathrm{a}-\mathrm{e}}$ \\
EGFRmAb.it & $104.50 \pm 14.66^{\mathrm{a}}$ & $41,146.04 \pm 9,966.85^{\mathrm{a}}$ \\
EGFRmAb-Au.it + NIR & $397.75 \pm 77.68^{\mathrm{a}, \mathrm{d}-\mathrm{f}}$ & $123,179.31 \pm 10,132.38^{\mathrm{a}, \mathrm{d}-\mathrm{f}}$ \\
\hline
\end{tabular}

Data are presented as the means \pm standard deviation. ${ }^{\mathrm{a}} \mathrm{P}<0.05$ compared with the NS .it group; ${ }^{\mathrm{b}} \mathrm{P}<0.05$ compared with the Au.it group; ${ }^{\mathrm{C}}<0.05$ compared with the Au.iv + NIR group; ${ }^{\mathrm{P}}<0.05$ compared with the NIR group; ${ }^{\mathrm{e}} \mathrm{P}<0.05$ compared with the EGFRmAb.it group; ${ }^{\mathrm{P}} \mathrm{P}<0.05$ compared with the Au.it + NIR group. Au, gold nanorods; EGFRmAb, epidermal growth factor monoclonal antibody; IOD, integrated optical density; it, intratumoral; iv, intravenous; NIR, near infrared spectroscopy; N.S., normal saline.

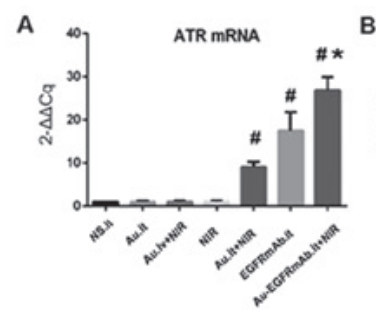

E

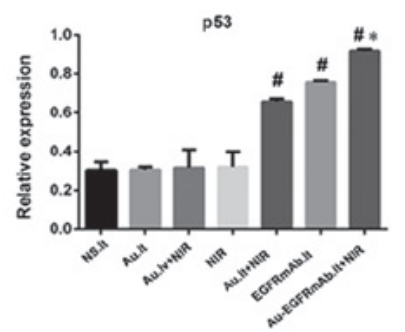

G

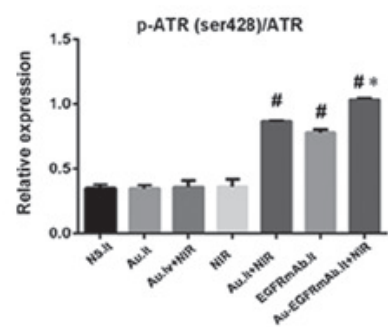

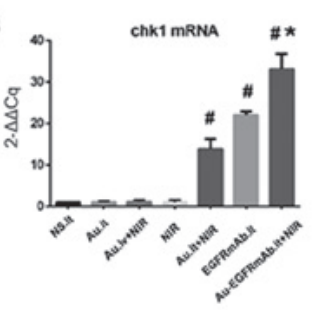

$\mathbf{F}$

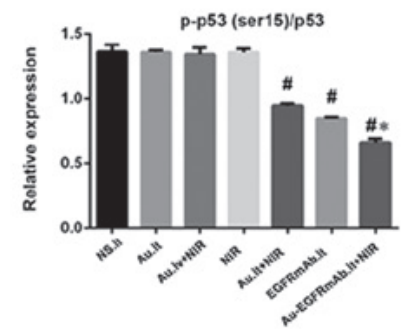

$\mathrm{H}$

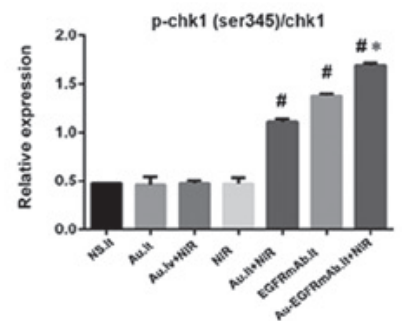

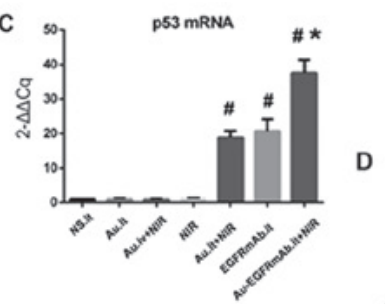

D
ATR

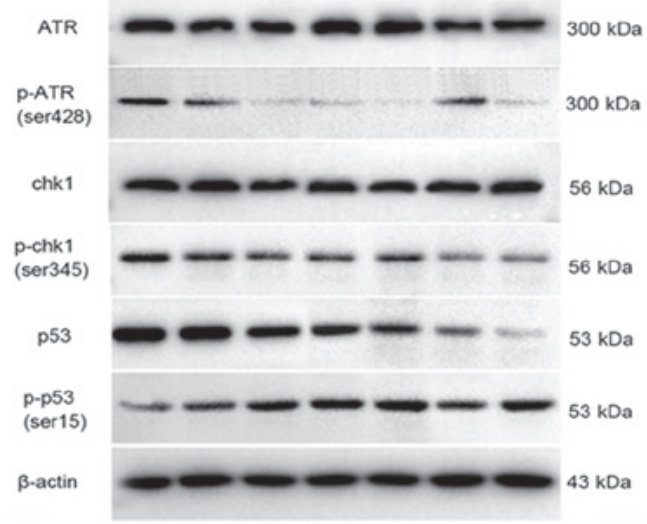

Figure 6. AuNRs plasmonic photothermal therapy activates the DNA damage signaling pathway, and EGFRmAb conjugation enhances the effect. (A-C) mRNA expression levels of ATR, Chk1 and p53. mRNA expression of each sample was detected by reverse transcription-quantitative polymerase chain reaction. The expression data of each group were normalized to the control group (N.S.it). (D-H) Protein expression levels of p-ATR, p-Chk1, p53 and p-p53. Relative protein expression levels are presented in the bar charts. $\beta$-actin was used as a control. ${ }^{~} \mathrm{P}<0.05$ compared with the NS.it group; ${ }^{*} \mathrm{P}<0.05$ compared with the Au.it $+\mathrm{NIR}$ and EGFRmAb.it groups. All experiments were repeated at least three times. ATR, ATR serine/threonine kinase; Au, gold nanorods; Chk1, checkpoint kinase 1; EGFRmAb, epidermal growth factor monoclonal antibody; it, intratumoral; iv, intravenous; NS, normal saline; NIR, near infrared spectroscopy; p, phosphorylated.

DNA damage pathway serves an important role in cell apoptosis; therefore, the present study aimed to determine whether it contributed to apoptosis triggered by AuNR-mediated PPTT in vivo. RT-qPCR was performed to determine the relative mRNA expression levels of key factors in this pathway, such as ATR, Chk1 and p53. For each gene, no obvious changes were observed among the control, AuNRs.it, AuNRs.iv + NIR and NIR groups (P>0.05; Fig. 6A-C). Compared with in the control group, the mRNA expression levels of all three genes were significantly augmented in mice treated with AuNRs.it + NIR, EGFRmAb. it or EGFRmAb-AuNRs.it + NIR (P<0.05; Fig. 6A-C). Furthermore, the EGFRmAb-AuNRs.it + NIR group exhibited the highest mRNA expression of all three genes, and the differences were statistically significant compared with the AuNRs. it + NIR and EGFRmAb.it groups $(\mathrm{P}<0.05$; Fig. 6A-C).

To further verify the effects of AuNRs.it + NIR, EGFRmAb.it and EGFRmAb-AuNRs.it + NIR on the DNA damage pathway, western blotting was performed to detect 
relative protein levels. As shown in Fig. 6D-H, the AuNRs. it, NIR and AuNRs.iv + NIR groups exhibited similar expression levels of all detected proteins compared with the control group $(\mathrm{P}>0.05)$. However, treatment with AuNRs.it + NIR, EGFRmAb.it and EGFRmAb-AuNRs.it + NIR significantly increased p-ATR, $\mathrm{p}-\mathrm{Chk} 1$ and $\mathrm{p} 53$ protein expression, but decreased $\mathrm{p}-\mathrm{p} 53$ expression $(\mathrm{P}<0.05)$. The most marked alterations were detected in the EGFRmAb-AuNRs.it + NIR group, and the differences were statistically significant compared with the AuNRs.it + NIR and the EGFRmAb.it groups $(\mathrm{P}<0.05$; Fig. $6 \mathrm{D}-\mathrm{H})$. These results suggested that EGFRmAb may synergistically interact with AuNRs to activate the DNA damage signaling pathway under NIR.

\section{Discussion}

$\mathrm{HC}$ is one of the most common malignant tumors of the head and neck, the survival rate of which is low due to difficulties in its diagnosis and surgery; therefore, the development of novel treatments for HC is essential. AuNRs absorb NIR and efficiently convert light into thermal energy. AuNR-mediated PPTT has been effectively adopted for the treatment of cancer in animal models; however, AuNRs alone have a poor specificity to tumor cells and a high cytotoxicity to normal tissues, as previously reported (6). It has been suggested that EGFR is overexpressed on the cell surface of several tumors, and EGFRmAb-AuNRs have exhibited enhanced specificity to cancer cells. For example, Durr et al (19) revealed that EGFRmAb-AuNRs specifically recognize epithelial cancer cells. In addition, Dickerson et al (20) constructed a mouse model of squamous carcinoma by injecting mice with human HSC-3 cells; the results revealed that, compared with in the control groups, EGFRmAb-AuNRs significantly inhibit tumor growth in vivo. These studies indicated that anti-EGFR antibodies may specifically target AuNRs to cancer cells and greatly improve the specificity of AuNR-induced PPTT; however, its effect on HC remains unclear.

The present study investigated the effects of EGFRmAbAuNRs on inhibiting tumor growth in BALB/C(nu/nu) nude mice, which are widely used for the study of malignant tumors. The effects of intravenous injection of AuNRs followed by NIR were tested; however, tumor growth was similar to that in the control group. This finding may be due to the outward growth phenomenon of tumor vessels and the first-pass elimination effect of the reticuloendothelial system; these factors may prevent the effective accumulation of AuNRs in tumor cells. Compared with intravenous administration, local treatment was more selective and effective; NIR triggered AuNR-mediated PPTT to prevent tumor growth in vivo. In addition, EGFRmAb conjugation was revealed to effectively target AuNRs to cancer cells and greatly enhance the inhibitory effects of AuNRs-mediated PPTT.

It was suggested that the reduced tumor size and weight caused by AuNR-mediated PPTT may be attributed to increased apoptosis. To investigate this hypothesis, flow cytometry and TUNEL experiments were performed separately, in order to detect apoptotic cells in isolated tumors. The results were consistent, compared with in the control group, the AuNRs.it + NIR, EGFRmAb. it and EGFRmAb-AuNRs.it + NIR groups exhibited increased apoptosis. In addition, the apoptotic rate in the EGFRmAb-AuNRs.it + NIR group was markedly higher than in the AuNRs.it + NIR and EGFRmAb.it groups. These observations indicated that EGFRmAb conjugation may enhance apoptosis induced by AuNRs + NIR, which in turn may contribute to reduced tumor growth in nude mice.

EGFR is a transmembrane tyrosine kinase receptor. It is well known that EGFR overexpression is associated with the occurrence and poor prognosis of numerous malignant tumors (21). EGFRmAb can block the binding of EGFR with epidermal growth factors, thus inhibiting downstream pathways activated by EGFR. Cetuximab is an EGFRmAb that targets the extracellular domain of EGFR (22); it has been used to treat head and neck cancer by specifically blocking the activation of EGFR downstream pathways. Bonner et al $(23,24)$ compared the effects of cetuximab + radiotherapy and radiotherapy alone on patients with advanced oropharyngeal and laryngeal cancer, and $\mathrm{HC}$. The results revealed that the survival rate of patients receiving cetuximab + radiotherapy is significantly higher than the rate of patients receiving radiotherapy only. In the present study, local injection of EGFRmAb into tumors induced apoptosis and decreased tumor growth. It is possible that EGFRmAb conjugation not only improves the specificity of AuNRs targeting to tumor cells, but also blocks EGFR-activated pathways, thus leading to the enhanced growth inhibitory effects of EGFRmAb-AuNRs.it + NIR.

The PI3K/Akt signaling pathway is activated by EGFR overexpression in various types of cancer and serves an important role in preventing apoptosis, promoting proliferation and increasing metastasis, etc. (25). PI3K/Akt pathway inhibitors, such as LY294002 and Wortmannin, have been used to treat cancer $(26,27)$. When the pathway is activated, phosphatidylinositol-3,4,5-trisphosphate is increased, which in turn phosphorylates Akt, the key factor of the pathway $(28,29)$.

Compared with in the control group, Akt levels were reduced in the AuNRs.it + NIR, EGFRmAb.it and EGFRmAb-AuNRs. it + NIR groups. Furthermore, the downstream factor GSK3 $\beta$ (30) was also decreased in these three groups. Notably, the most marked alterations in Akt and GSK3 $\beta$ expression occurred in the EGFRmAb-AuNRs.it + NIR group. To further confirm the inhibition of the PI3K/Akt pathway, the present study determined the effects of various treatments on PTEN, which is a negative regulator of the PI3K/Akt pathway $(31,32)$. PTEN mRNA and protein levels were significantly augmented in the AuNRs.it + NIR, EGFRmAb.it and EGFRmAb-AuNRs.it + NIR groups; the increase was markedly increased in the EGFRmAb-AuNRs.it + NIR group compared with in the other two groups. Taken together, AuNRs.it + NIR may downregulate the PI3K/Akt signaling pathway by decreasing Akt and GSK3 $\beta$, as well as increasing PTEN. EGFRmAb conjugation further increased the inhibition of this pathway.

When DNA damage cannot be repaired in a timely manner, apoptosis occurs. Clinically, chemotherapy and radiotherapy cause serious DNA damage to tumor cells, inducing cell apoptosis and ultimately killing the tumor cells (33). Therefore, the present study investigated the role of the DNA damage pathway in AuNRs-mediated tumor inhibition. ATR is a key factor of this pathway. In response to DNA damage, ATR is phosphorylated, consequently phosphorylating Chk1 and activating p53 to induce cell death (34). In the present study, the 
mRNA and protein expression levels of ATR, Chk1 and p53 were detected. The results indicated that AuNRs augmented the mRNA expression of all three genes, and EGFRmAb conjugation further enhanced mRNA expression, as compared with single treatments using AuNRs or EGFRmAb. Furthermore, all three local treatments (AuNRs.it + NIR, EGFRmAb.it and EGFRmAb-AuNRs.it + NIR) significantly upregulated p-ATR, p-Chk1 and p53. Similar with other results, the most marked increase was observed in the EGFRmAb-AuNRs.it + NIR group. Overall, AuNRs.it + NIR may trigger apoptosis partially by activating the DNA damage signaling pathway, and EGFRmAb conjugation further increased the effect. AuNR-based PPTT has shown significant promise for the selective ablation of cancer cells (35). It has been reported that AuNRs act as efficient PPTT agents that induce cell death through hyperthermia while scavenging the reactive oxygen species (ROS) produced during treatment (35). ROS can potentially interact with neighboring healthy tissues, causing irreversible damage to DNA (36).

There were some limitations to the present study. Firstly, tumor targeting was not investigated, since administrations were intratumoral. Secondly, analysis of the associated signaling pathway was insufficient, as other signaling pathways, including oxidative stress pathways, were not investigated. Therefore, further study on the underlying mechanism is required.

In conclusion, the present study revealed that EGFRmAb conjugation enhanced the inhibitory effects of AuNRs-mediated PPTT on tumor growth in a mouse model of HC. Based on our previous studies and the present study, EGFRmAb may target AuNRs to tumor cells by binding to EGFR. The enhanced specificity of AuNRs increased their inhibitory effects on tumor growth. In addition, EGFRmAb conjugation may also increase tumor cell apoptosis by downregulating the PI3K/Akt pathway and upregulating the DNA damage pathway. These results provided novel insights into the effects of targeted PPTT on cancer treatment, particularly HC treatment.

\section{Acknowledgements}

The authors would like to thank the Kunming Institute of Precious Metals (Kunming, China) and the National Center for Nanoscience and Technology (Kunming, China) for technical support during the study.

\section{Funding}

This study was supported by the National Natural Science Foundation of China (grant no. 81160324).

\section{Availability of data and materials}

The datasets used and/or analyzed during the current study are available from the corresponding author on reasonable request.

\section{Authors' contributions}

YZh analyzed the experimental data, perfomed in vitro experiments and was a major contributor in writing the manuscript. JH analyzed the experimental data. YW and JW performed the in vivo experiments; $\mathrm{YZo}$ and $\mathrm{ZY}$ performed the in vitro experiments. XH designed the study and provided the research funds.

\section{Ethics approval and consent to participate}

All experimental procedures were conducted in accordance with the institutional guidelines for the care and use of laboratory animals. The present study was approved by the Institutional Animal Care and Use Committee of Kunming Medical University.

\section{Patient consent for publication}

Not applicable.

\section{Competing interests}

The authors declare that they have no competing interests.

\section{References}

1. Rivière D, Mancini J, Santini L, Giovanni A, Dessi P and Fakhry N: Lymph-node metastasis following total laryngectomy and total pharyngolaryngectomy for laryngeal and hypopharyngeal squamous cell carcinoma: Frequency, distribution and risk factors. Eur Ann Otorhinolaryngol Head Neck Dis 135: 163-166, 2018.

2. Krstevska V, Stojkovski I and Lukarski D: Concurrent radiochemotherapy in advanced hypopharyngeal cancer. Radiat Oncol 5: 39, 2010.

3. Cabral RM and Baptista PV: Anti-cancer precision theranostics: A focus on multifunctional gold nanoparticles. Expert Rev Mol Diagn 14: 1041-1052, 2014.

4. Aioub M and El-Sayed MA: A real-time surface enhanced raman spectroscopy study of plasmonic photothermal cell death using targeted gold nanoparticles. J Am Chem Soc 138: 1258-1264, 2016.

5. Brand TM, Iida M, Li C and Wheeler DL: The nuclear epidermal growth factor receptor signaling network and its role in cancer. Discov Med 12: 419-432, 2011.

6. Zhang S, Li Y, He X, Dong S, Huang Y, Li X, Li Y, Jin C, Zhang Y and Wang Y: Photothermolysis mediated by gold nanorods modified with EGFR monoclonal antibody induces Hep-2 cells apoptosis in vitro and in vivo. Int J Nanomedicine 9: 1931-1946, 2014.

7. Normanno N, De Luca A, Bianco C, Strizzi L, Mancino M, Maiello MR, Carotenuto A, De Feo G, Caponigro F and Salomon DS: Epidermal growth factor receptor (EGFR) signaling in cancer. Gene 366: 2-16, 2006.

8. Garrett TP, McKern NM, Lou M, Elleman TC, Adams TE, Lovrecz GO, Zhu HJ, Walker F, Frenkel MJ, Hoyne PA, et al: Crystal structure of a truncated epidermal growth factor receptor extracellular domain bound to transforming growth factor alpha. Cell 110: 763-773, 2002.

9. Sourbier C, Lindner V, Lang H, Agouni A, Schordan E, Danilin S, Rothhut S, Jacqmin D, Helwig JJ and Massfelder T: The phosphoinositide 3-kinase/Akt pathway: A new target in human renal cell carcinoma therapy. Cancer Res 66: 5130-5142, 2006.

10. Hotfilder M, Sondermann P, Senss A, van Valen F, Jürgens H and Vormoor J: PI3K/AKT is involved in mediating survival signals that rescue Ewing tumour cells from fibroblast growth factor 2-induced cell death. Br J Cancer 92: 705-710, 2005.

11. Sandra CM, Eduardo CC, Simon HO, Teresa RA, Antonio NC, Lijanova IV and Marcos MG: Anticancer activity and antiinflammatory studies of 5-aryl-1,4-benzodiazepine derivatives. Anticancer Agents Med Chem 12: 611-618, 2012.

12. Clemens MJ: Targets and mechanisms for the regulation of translation in malignant transformation. Oncogene 23: 3180-3188, 2004.

13. Vignard J, Mirey G and Salles B: Ionizing-radiation induced DNA double-strand breaks: A direct and indirect lighting up. Radiother Oncol 108: 362-369, 2013. 
14. Ciccia A and Elledge SJ: The DNA damage response: Making it safe to play with knives. Mol Cell 40: 179-204, 2010.

15. Abraham RT: Cell cycle checkpoint signaling through the ATM and ATR kinases. Genes Dev 15: 2177-2196, 2001.

16. Demoulin B, Hermant M, Castrogiovanni C, Staudt C and Dumont P: Resveratrol induces DNA damage in colon cancer cells by poisoning topoisomerase II and activates the ATM kinase to trigger p53-dependent apoptosis. Toxicol In Vitro 29: $1156-1165,2015$

17. Zhang Y, Cong L, He J, Wang Y, Zou Y, Yang Z, Hu Y, Zhang S and $\mathrm{He} \mathrm{X}$ : Photothermal treatment with EGFRmAb-AuNPs induces apoptosis in hypopharyngeal carcinoma cells via $\mathrm{PI} 3 \mathrm{~K} / \mathrm{AKT} / \mathrm{mTOR}$ and DNA damage response pathways. Acta Biochim Biophys Sin (Shanghai) 50: 567-578, 2018.

18. Livak KJ and Schmittgen TD: Analysis of relative gene expression data using real-time quantitative PCR and the 2(-Delta Delta C(T)) Method. Methods 25: 402-408, 2001.

19. Durr NJ, Larson T, Smith DK, Korgel BA, Sokolov K and Ben-Yakar A: Two-photon luminescence imaging of cancer cells using molecularly targeted gold nanorods. Nano Lett 7: 941-945, 2007.

20. Dickerson EB, Dreaden EC, Huang X, El-Sayed IH, Chu H, Pushpanketh S, McDonald JF and El-Sayed MA: Gold nanorod assisted near-infrared plasmonic photothermal therapy (PPTT) of squamous cell carcinoma in mice. Cancer Lett 269: 57-66, 2008.

21. Castellani R, Visscher DW, Wykes S, Sarkar FH and Crissman JD: Interaction of transforming growth factor-alpha and epidermal growth factor receptor in breast carcinoma. An immunohistologic study. Cancer 73: 344-349, 1994.

22. Giampieri R, Scartozzi M, Del Prete M, Maccaroni E, Bittoni A, Faloppi L, Bianconi M, Cecchini L and Cascinu S: Molecular biomarkers of resistance to anti-EGFR treatment in metastatic colorectal cancer, from classical to innovation. Crit Rev Oncol Hematol 88: 272-283, 2013.

23. Bonner JA, Harari PM, Giralt J, Azarnia N, Shin DM, Cohen RB, Jones CU, Sur R, Raben D, Jassem J, et al: Radiotherapy plus cetuximab for squamous-cell carcinoma of the head and neck. N Engl J Med 354: 567-578, 2006.

24. Bonner JA, Harari PM, Giralt J, Cohen RB, Jones CU, Sur RK, Raben D, Baselga J, Spencer SA, Zhu J, et al: Radiotherapy plus cetuximab for locoregionally advanced head and neck cancer: 5 -year survival data from a phase 3 randomised trial, and relation between cetuximab-induced rash and survival. Lancet Oncol 11: $21-28,2010$.
25. Ahlmann E, Patzakis M, Roidis N, Shepherd L and Holtom P: Comparison of anterior and posterior iliac crest bone grafts in terms of harvest-site morbidity and functional outcomes. J Bone Joint Surg Am. 84-A: 716-720, 2002.

26. LoPiccolo J, Blumenthal GM, Bernstein WB and Dennis PA: Targeting the PI3K/Akt/mTOR pathway: effective combinations and clinical considerations. Drug Resist Updat 11: 32-50, 2008.

27. Catasus L, D'Angelo E, Pons C, Espinosa I and Prat J: Expression profiling of 22 genes involved in the PI3K-AKT pathway identifies two subgroups of high-grade endometrial carcinomas with different molecular alterations. Mod Pathol 23: 694-702, 2010.

28. Priulla M, Calastretti A, Bruno P, Azzariti A, Paradiso A, Canti G and Nicolin A: Preferential chemosensitization of PTEN-mutated prostate cells by silencing the Akt kinase. Prostate 67: 782-789, 2007.

29. Zhang D and Fan D: Multidrug resistance in gastric cancer: Recent research advances and ongoing therapeutic challenges. Expert Rev Anticancer Ther 7: 1369-1378, 2007.

30. Yang L, Dan HC, Sun M, Liu Q, Sun XM, Feldman RI, Hamilton AD, Polokoff M, Nicosia SV, Herlyn M, et al: Akt/protein kinase B signaling inhibitor-2, a selective small molecule inhibitor of Akt signaling with antitumor activity in cancer cells overexpressing Akt. Cancer Res 64: 4394-4399, 2004.

31. Liu X, Dai X and Wu B: Study of 5-Aza-CdR on transcription regulation of RASSF1A gene in the BIU87 cell line. Urol Int 82: 108-112, 2009.

32. Liao W, McNutt MA and Zhu WG: The comet assay: A sensitive method for detecting DNA damage in individual cells. Methods 48: 46-53, 2009.

33. Lieberman HB: DNA damage repair and response proteins as targets for cancer therapy. Curr Med Chem 15: 360-367, 2008.

34. Matsumoto K, Nagahara T, Okano J and Murawaki Y: The growth inhibition of hepatocellular and cholangiocellular carcinoma cells by gemcitabine and the roles of extracellular signal-regulated and checkpoint kinases. Oncol Rep 20: 863-872, 2008.

35. Aioub M, Panikkanvalappil SR and El-Sayed MA: Platinumcoated gold nanorods: Efficient reactive oxygen scavengers that prevent oxidative damage toward healthy, untreated cells during plasmonic photothermal therapy. ACS Nano 11: 579-586, 2017.

36. Schieber M and Chandel NS: ROS function in redox signaling and oxidative stress. Curr Biol 24: R453-R462, 2014. 